\title{
Optically switchable mirrors for surface plasmon polaritons propagating on semiconductor surfaces
}

\author{
J. Gómez Rivas, ${ }^{1,2}$ J. A. Sánchez-Gil,$^{3}$ M. Kuttge, ${ }^{1}$ P. Haring Bolivar,,${ }^{1,4}$ and H. Kurz ${ }^{1}$ \\ ${ }^{1}$ Institut für Halbleitertechnik, RWTH-Aachen University, Sommerfeldstraße 24, D-52074 Aachen, Germany \\ ${ }^{2}$ Center for Nanophotonics, FOM Institute AMOLF, c/o Philips Research Laboratories, \\ High Tech Campus 4, 5656 AE Eindhoven, The Netherlands \\ ${ }^{3}$ Instituto de Estructura de la Materia, Consejo Superior de Investigaciones Científicas, Serrano 121, E-28006 Madrid, Spain \\ ${ }^{4}$ Institute of High Frequency and Quantum Electronics, University of Siegen, Hölderlinstrasse 3, D-57068 Siegen, Germany
}

(Received 21 March 2006; revised manuscript received 26 July 2006; published 20 December 2006)

\begin{abstract}
Using terahertz time-domain spectroscopy measurements, we demonstrate optical switching of the transmission of terahertz surface plasmon polaritons (SPPs) propagating on an indium antimonide surface through Bragg scattering on grooves. The switching of the in-plane transmission of SPPs through the grooves is mediated by the modification of the stop-gap width due to changes of the surface plasmon characteristics, which are induced by the optical generation of charge carriers. The use of a semiconductor surface allows us to achieve switching at extremely low excitation powers. The experimental data can be quantitatively reproduced using rigorous numerical calculations for surfaces with varying dielectric function. Such an optically active device opens further possibilities for low-frequency surface plasmon optics.
\end{abstract}

DOI: 10.1103/PhysRevB.74.245324

PACS number(s): 73.20.Mf, 42.79.Dj, 42.25.Bs, 78.68.+m

\section{INTRODUCTION}

Surface plasmon polaritons (SPPs) are electromagnetic waves coupled to the coherent oscillation of free charge carriers at the interface between a dielectric and a conductor. ${ }^{1}$ SPPs are often referred to as low-dimensional waves due to the strong confinement of the electromagnetic field to the surface. $^{2}$ This remarkable property, which allows twodimensional optics below the diffraction limit, has been the reason for a great increase of interest in the field of surface plasmon optics over the past few years, which has led to the observation of a wealth of new phenomena. ${ }^{3-8}$ In order to fully exploit the possibilities of plasmonic devices, it is necessary to modify the propagation of SPPs. In this regard, a great challenge in the field of plasmonics or surface plasmon optics is the active control of surface plasmon polaritons. It has been demonstrated that the efficiency with which light is coupled into a gallium surface plasmon polariton wave guide can be optically controlled by a nanoscale light-induced structural transformation in the Ga layer. ${ }^{9}$ Thermo-optical control of the propagation of SPPs propagating on gold stripes embedded in polymer was recently demonstrated. ${ }^{10,11}$ Electrochemical switching between the reduced and the oxidized states of conducting polymers have been proposed as a mean for active plasmonics with metals coated with polymers. ${ }^{12}$ The SPP-assisted transmission of light through subwavelength apertures, ${ }^{13}$ arrays of apertures, ${ }^{14,15}$ and slits ${ }^{16}$ can be actively controlled when combined with nonlinear materials. Semiconductors are efficient materials for active plasmonics in the terahertz $(\mathrm{THz})$ frequency regime. Thermal and optical switching of the transmission of $\mathrm{THz}$ radiation through arrays of subwavelength apertures have been recently demonstrated. ${ }^{17,18}$ For in-plane propagation of $\mathrm{THz}$ SPPs on semiconductor surfaces, it has been demonstrated that the SPP transmission across Bragg mirrors can be suppressed thermally. ${ }^{19,20}$

Here, we demonstrate the optical modification of the propagation of $\mathrm{THz}$ SPPs on a semiconductor surface. We study the in-plane SPP propagation and scattering on arrays of grooves of size comparable to the wavelength. In contrast to previous works on optically active $\mathrm{THz}$ plasmonics, where the transmission through arrays of subwavelength holes is investigated, ${ }^{18}$ we present here a demonstration of the optical control of the in-plane propagation of THz SPPs. It is shown that the differential transmission of SPPs through a semiconductor grating or Bragg mirror, consisting of 30 grooves structured in undoped InSb, can be increased by as much as almost a factor 15 at certain frequencies by the optical excitation of free carriers and the consequent modification of the dielectric properties of the surface. This modification gives rise to a narrowing of the stop gap that results from the SPP Bragg scattering. The increase of the differential transmission is well reproduced by numerical calculations and it is in contrast with the reduction of the $\mathrm{THz}$ transmission through arrays of holes with optical pump attributed to the decrease of the effective hole size. ${ }^{18}$ A semiconductor all-optical switch for SPPs like the one presented here will offer the possibility of ultrafast active plasmonics at extremely low pumping fluences, i.e., 4-5 orders of magnitude lower than with similar metallic structures. Moreover, the modification of the SPPs characteristics takes place only on the illuminated area, providing a higher control of the SPPs propagation than with thermally active devices. ${ }^{19}$

The propagation of SPPs and their characteristic decay lengths away from the surface depend on the permittivities of the dielectric and the conductor. For an air-conductor interface, the SPPs decay length into air varies with the square root of the absolute value of the conductor's permittivity, ${ }^{1}$ which in the Drude approximation is given by $\varepsilon \simeq 1-\nu_{p}^{2} / \nu^{2}$. The plasma frequency $\nu_{p}$ is equal to $\nu_{p}$ $=(1 / 2 \pi) \sqrt{N e^{2} / m^{*} \varepsilon_{\infty} \varepsilon_{0}}$, where $N$ is the free carrier density, $e$ the electron charge, $m^{*}$ the carrier effective mass, and $\varepsilon_{\infty}$ and $\varepsilon_{0}$ the high-frequency and vacuum permittivities, respectively. The large carrier density in metals $\left(N \simeq 10^{23} \mathrm{~cm}^{-3}\right)$ leads to $\nu_{p} \simeq 1000 \mathrm{THz}$, i.e., in the uv or in the visible part of 
(a)

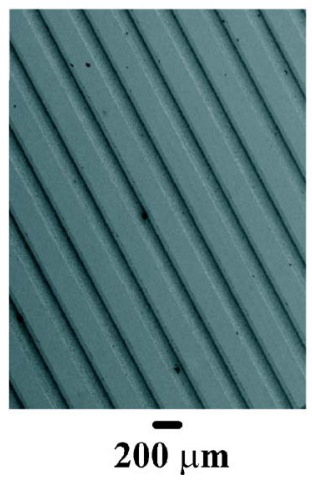

(b)

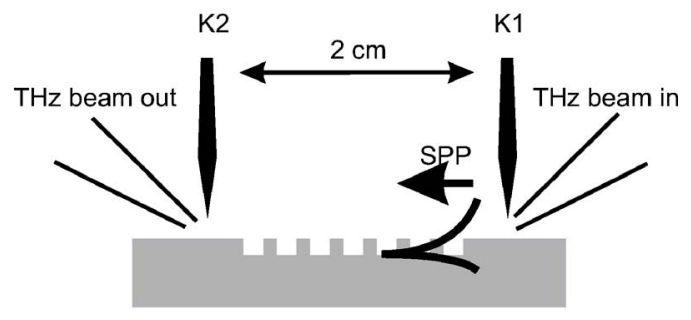

Schematic
FIG. 1. (Color online) (a) Scanning electron microscope top view of a SPP Bragg mirror or grating structured on InSb. (b) Schematic representation of the SPP coupling section of the experimental setup. Free space $\mathrm{THz}$ radiation is scattered at the aperture defined by a razor blade and the surface of the sample coupling to SPPs. After transmission through the Bragg mirror, the SPPs are coupled back to free space $\mathrm{THz}$ radiation. the electromagnetic spectrum. The optical permittivity of metals thus has a small absolute value and SPPs have a short decay length $(\simeq 100 \mathrm{~nm})$ into air in the visible part of the electromagnetic spectrum. However, the absolute value of the permittivity varies with $\nu^{-2}$, reaching values as high as $|\varepsilon| \simeq 10^{6}$ at terahertz and microwave frequencies. These large values of the permittivity lead to huge SPP decay length into air on the order of several centimeters. Recently, complex metallic structures with a low effective plasma frequency have been proposed. ${ }^{21,22}$ These structures allow the use of metals for low-frequency plasmonics with a large enhancement of the electromagnetic field at the surface. ${ }^{23}$ On the other hand, semiconductors are good alternatives to metals for the excitation of low-frequency SPPs. ${ }^{24}$ The carrier concentration in semiconductors is determined by their dopant density and the intrinsic carrier concentration, being several orders of magnitude lower than that of metals. Consequently, the permittivity of semiconductors at terahertz and microwave frequencies is comparable to that of metals at optical frequencies, and subwavelength SPPs decay lengths into air can be achieved at low frequencies on semiconductor surfaces. A decisive advantage of semiconductors is that their carrier density and mobility, and consequently the SPP characteristics, can be easily controlled by optical, thermal, or electrical injection of free carriers. As the carrier densities in semiconductors are much lower than those in metals, active semiconductor plasmonic elements are expected to be significantly more efficient, operating at several orders of magnitude lower carrier modulation densities.

This paper is organized as follows. In Sec. II the fabrication and characteristics of the SPP Bragg reflector are described together with a brief discussion of the experimental setup. The optical switching experiments of the in-plane transmission of $\mathrm{THz}$ SPPs through the Bragg reflector are presented in Sec. III. In Sec. III A the dependence of the switching of the transmission on the optical pump power is discussed. Subsequently, the dependence of the switching with the temperature of the semiconductor is investigated in Sec. III B. A discussion on dynamical switching is presented in Sec. IV. The paper ends with some concluding remarks.

\section{SAMPLES AND EXPERIMENTAL SETUP}

The large wavelength of $\mathrm{THz}$ radiation allows for the straightforward mechanical fabrication of resonant devices at these frequencies. The investigated grating was prepared by dicing 30 parallel cuts with a wafer saw (Disco DAD 321) on a 2 in. wafer of intrinsic InSb. The width of the cuts is $221 \mu \mathrm{m}$ and the depth $70 \mu \mathrm{m}$. The spacing between consecutive cuts is $221 \mu \mathrm{m}$, defining a lattice constant of $442 \mu \mathrm{m}$. A scanning electron microscope top view of the grating is shown in Fig. 1(a). InSb has a small energy gap of $0.17 \mathrm{eV}$. At room temperature the intrinsic carrier concentration of undoped $\mathrm{InSb}$ is $\simeq 10^{16} \mathrm{~cm}^{-3}$ and the corresponding $\nu_{\mathrm{p}}$ lies around $1.9 \mathrm{THz}$.

We use a THz-time domain spectrometer to excite SPP pulses on an InSb surface and to measure their propagation and transmission across the grating. ${ }^{25}$ In such a setup a train of pulses from Ti:sapphire laser is split into two beams. One of these beams constitutes the excitation beam while the other is the probe beam. The excitation pulses are used to optically generate coherent broadband $\mathrm{THz}$ radiation from an InGaAs surface field emitter and the probe pulses gate a photoconductive antenna as receiver. By varying the time delay between the two pulse trains, the $\mathrm{THz}$ pulse amplitude can be detected as a function of time with subpicosecond resolution. We use the aperture excitation technique to couple the free space $\mathrm{THz}$ radiation to SPPs and to couple back SPPs to free space $\mathrm{THz}$ radiation after propagating on the surface of the semiconductor. ${ }^{26}$ This technique has the important advantage that it allows broadband coupling. Figure 1(b) illustrates the SPP coupling section of our experimental setup, including SPP incoupling, SPP propagation along the Bragg reflector, and SPP outcoupling. $\mathrm{THz}$ radiation is focused into a small gap defined by the edge of a razor blade, denoted by K1, and the sample surface. The size of the gap is $300 \mu \mathrm{m}$. This geometry leads to scattering of the incident radiation and the generation of evanescent waves comprising a continuum of wave vectors. As a consequence, the dispersion relations of a fraction of the incident $\mathrm{THz}$ radiation and the SPPs match, i.e., coupling becomes possible. The excited SPPs propagate onto the InSb surface and impinge on the grating structured in the wafer. The transmitted SPPs through the grating are coupled back into free space $\mathrm{THz}$ radiation with another razor blade $\mathrm{K} 2$ placed at $300 \mu \mathrm{m}$ above the sample surface and at a distance of $2 \mathrm{~cm}$ from $\mathrm{K} 1$. The outcoupled $\mathrm{THz}$ radiation is then guided to the detection antenna. As will be discussed later, reducing the temperature of the grating diminish the density of thermally excited carriers and enhances the optical switching efficiency. There- 

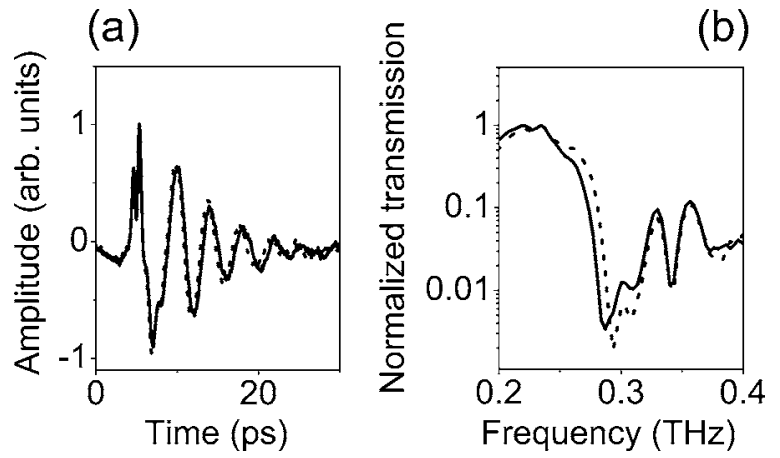

FIG. 2. (a) SPP pulse transmitted through an InSb grating with a lattice constant of $421 \mu \mathrm{m}$ formed by 30 grooves with a width of $212 \mu \mathrm{m}$ and a depth of $70 \mu \mathrm{m}$ at a temperature of $-35^{\circ} \mathrm{C}$ (solid line). The dotted line represents a SPP pulse propagating through the grating illuminated with an optical pump $\left(7 \mathrm{~W} / \mathrm{cm}^{2}\right)$. (b) Normalized transmission spectra of SPPs through the grating at a temperature of $-35{ }^{\circ} \mathrm{C}$ without optical pump (solid line) and with an optical pump density of $7 \mathrm{~W} / \mathrm{cm}^{2}$ (dotted line).

fore, the grating was placed on top of a thermoelectric cooler at $-35^{\circ} \mathrm{C}$ (not shown on Fig. 1). At this temperature the density of thermally excited carriers is reduced to $N \simeq 0.3$ $\times 10^{16} \mathrm{~cm}^{-3}$ and the plasma frequency shifts to $\nu_{p} \simeq 1 \mathrm{THz}$. These values of $N$ and $\nu_{p}$ were determined by measuring the $\mathrm{THz}$ transmission through a piece of $\mathrm{InSb}$ as described in Ref. 28. The black line in Fig. 2(a) represents a SPP pulse after propagating through the grating at $-35^{\circ} \mathrm{C}$.

To analyze the spectral dependence of the transmission, the measured $\mathrm{THz}$ pulses were Fourier transformed to obtain the transmission power spectra. The solid line in Fig. 2(b) represents the normalized transmission through the grating at $-35^{\circ} \mathrm{C}$. Bragg scattering of SPPs by the periodic structure opens a stop gap around $\nu=0.3 \mathrm{THz}$, where the transmission is remarkably reduced by almost three orders of magnitude. The interference of SPPs scattered at different grooves are responsible for the drastic reduction of the transmittance in this frequency range. The transmission does not reach unity at high frequencies mainly due to radiative leakage induced by the grating. ${ }^{27}$

\section{OPTICAL SWITCHING OF THE IN-PLANE TRANSMISSION OF SPPS THROUGH A BRAGG MIRROR}

In this section the experiments on the optical switching of the transmission of SPPs through the grating and the comparison with the calculations are presented. In Sec. III A the dependence of the switching on the pump power is discussed. The impact of the temperature of the sample on the switching efficiency is presented in Sec. III B.

We used an expanded beam with a $1 \mathrm{~cm}$ diameter from a single-mode $\mathrm{cw}$ ytterbium fiber laser with a wavelength of $1075 \mathrm{~nm}$ to photoexcite carriers and modulate the SPP properties. The dotted lines in Figs. 2(a) and 2(b) represent a SPP pulse transmitted through the grating and the normalized SPP transmission through the grating illuminated with an optical power of $7 \mathrm{~W} / \mathrm{cm}^{2}$. A low absolute value of the real part of permittivity of the conductor leads to efficient scat-

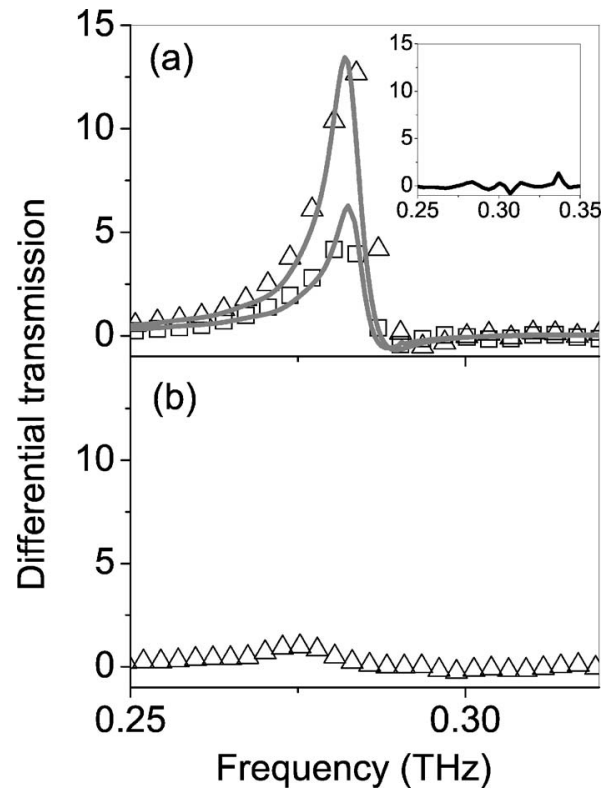

FIG. 3. (a) Differential SPP transmission through a grating of $\mathrm{InSb}$ at a temperature of $-35^{\circ} \mathrm{C}$ illuminated by a laser beam with a diameter of $1 \mathrm{~cm}$ and $\lambda=1075 \mathrm{~nm}$. The squares correspond to a pumping power of $4.5 \mathrm{~W} / \mathrm{cm}^{2}$, while the triangles to $7 \mathrm{~W} / \mathrm{cm}^{2}$. The lines are fits to the measurements. The inset shows the same ratio for a pumping power of $7 \mathrm{~W} / \mathrm{cm}^{2}$ but with the laser beam displaced one beam diameter away from the path of the SPPs. (b) Differential transmission through the same grating as in (a) illuminated by a laser beam with $\lambda=532 \mathrm{~nm}$ and $P=6.4 \mathrm{~W} / \mathrm{cm}^{2}$.

tering of SPPs at the grooves and to a wide stop gap for SPPs. The optical pump creates an electron-hole plasma at the surface of the grating, shifting the plasma frequency to higher values. As the pump power is increased, more free carriers are excited and the shifts becomes more pronounced, leading to a larger absolute value of the permittivity at $\mathrm{THz}$ frequencies and to a weaker confinement of the SPPs to the surface. As a consequence of this weaker confinement, the scattering of SPPs becomes less intense and the gap narrows. ${ }^{29-31}$

\section{A. Pump power dependence of the switching}

The differential SPP transmission, defined as $\left(T-T_{0}\right) / T_{0}$, where $T$ is the transmission through the grating and $T_{0}$ is the transmission without optical pump, of SPPs measured for different power densities $P$ of the laser are represented in Fig. 3(a). This transmission ratio represents the optical switching efficiency of the grating. The squares and triangles of Fig. 3(a) correspond to the differential transmissions for $P=4.5$ and $7 \mathrm{~W} / \mathrm{cm}^{2}$, respectively. As the power of the laser is increased, the switching efficiency becomes larger. For power densities as low as $7 \mathrm{~W} / \mathrm{cm}^{2}$ the differential transmission experiences a 13-fold increase at $0.28 \mathrm{THz}$ with respect to the transmission without optical pump. The increase of the differential transmission of Fig. 3(a) is thus the consequence of the shift of the lower band edge of the gap due to its narrowing. 
The effect of the optical pump on the SPP transmission is similar to heating the grating, ${ }^{19,20}$ but it should be stressed that the carriers are now generated optically. To rule out thermal effects on the optical switching measurements, we verified that the temperature increase from no pumping to maximum pumping power was smaller than $5^{\circ} \mathrm{C}$. For this temperature difference no significant changes on the $\mathrm{THz}$ transmission through the grating occur.

We repeated the optical switching experiment using a single-mode cw diode-pumped laser with a wavelength of $532 \mathrm{~nm}$. These measurements are shown in Fig. 3(b), where the triangles correspond to the differential transmission for $P=6.4 \mathrm{~W} / \mathrm{cm}^{2}$. The change on the differential transmission is much less pronounced than with a pump of $\lambda=1075 \mathrm{~nm}$. Note that the scales of Figs. 3(a) and 3(b) are the same for direct comparison. The lower switching efficiency with a pump of $\lambda=532 \mathrm{~nm}$ for a similar pumping power results from the lower carrier density excited due to the higher photon energy at this $\lambda$. As thermal effects would show only a minimal dependence with the excitation wavelength, the different switching observed further underscores the nonthermal character of the switching effect for $\lambda=1075 \mathrm{~nm}$.

The local character of the optical switching, i.e., SPP scattering is only modified on the area under illumination, was verified by measuring the transmission of SPPs through the grating for $P=7 \mathrm{~W} / \mathrm{cm}^{2}$ with the laser beam displaced one beam diameter away from the SPP path. These measurements are displayed in the inset of Fig. 3 using the same scale as in the main figure for direct comparison. As expected, there is no significant change on the transmission with and without illumination when the pump beam does not intercept the path of SPPs. This measurement also confirms that the switching of the transmission is not due to heating the sample.

We have estimated an average plasma frequency $\overline{\nu_{p}}$ of the illuminated grating with $\lambda=1075 \mathrm{~nm}$ as follows: We simplify the problem by considering the grating as a homogeneous medium, without spatial variation of its permittivity or its plasma frequency. The spatially non-uniform illumination of the grating and the inhomogeneous distribution of optically generated carriers beneath the surface complicate greatly the exact calculation of the scattering characteristics of our system, and forces us to use the simplification. Under this assumption we can calculate the differential SPP transmission through the grating for different pumping powers employing the rigorous electromagnetic formulation of the Green's theorem surface integral equations; ${ }^{32}$ this formulation is applied to a scattering geometry resembling the experimental configuration. ${ }^{33}$ The frequency dependence of the intensity of the SPP transmitted through the groove array is obtained from the electromagnetic field intensity at the position where the SPPs are coupled back to electromagnetic radiation, normalized by that obtained in the absence of the groove array (flat surface). This is done for different values of the plasma frequency of the substrate, yielding several SPP transmission spectra which are subsequently normalized by the reference spectrum in the absence of optical pumping (for a known plasma frequency determined by the temperature). The resulting curves are compared to the experimental data, thus obtaining the average plasma frequencies from the best fits (a)

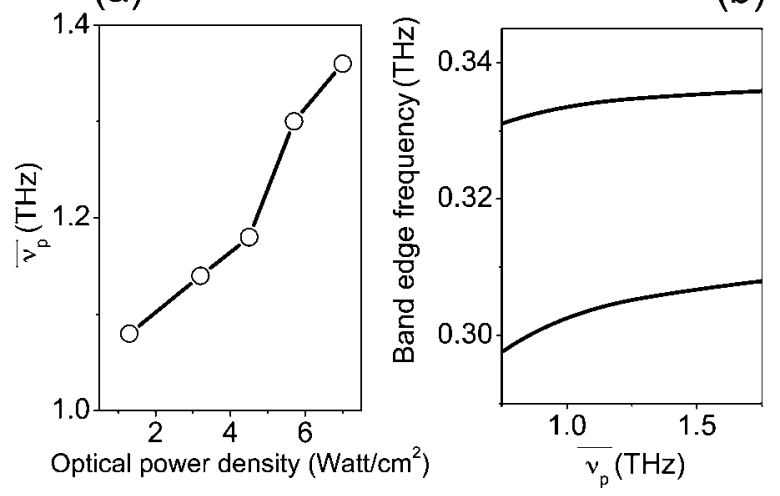

FIG. 4. (a) Average plasma frequency of the InSb grating illuminated by a laser beam with a diameter of $1 \mathrm{~cm}$ and $\lambda$ $=1075 \mathrm{~nm}$ as a function of the optical power. (b) Dependence of the lower and upper band edges on the average plasma frequency.

[solid lines in Fig. 3(a)]:34 $\overline{\nu_{p}}=1.2$ and $1.4 \mathrm{THz}$ for $P=4.5$ and $7 \mathrm{~W} / \mathrm{cm}^{2}$, respectively.

The average plasma frequencies at these and intermediate pumping power densities are represented in Fig. 4(a), where the solid line is a guide to the eye. As discussed above, the increase of $P$ excites more free charge carriers shifting the plasma frequency to larger values. The impact of varying $\overline{\nu_{p}}$ on the first SPP band gap is shown in Fig. 4(b), on the basis of the following approximate analytical expression for the lower and upper band edges ${ }^{20}$

$$
\frac{\omega_{ \pm}\left(\overline{\nu_{p}}\right)}{c} \approx \frac{G}{2}\left(1-\frac{1}{\varepsilon\left(\overline{\nu_{p}}\right)}\left[1+s_{0}\left(\overline{\overline{\nu_{p}}}\right) f_{0} \mp\left|s_{0}\left(\overline{\nu_{p}}\right) f_{1}\right|\right]^{2}\right)^{-1 / 2},
$$

where $G$ is the reciprocal lattice vector and $c$ the speed of light in vacuum. This expression is obtained to lowest order in the surface impedance and restricted to the first SPP Bragg scattering [with $s_{0} \equiv(1-\varepsilon)\left(1-\delta^{2}\right)^{1 / 2} /(\delta \varepsilon)$, omitting the $\nu_{p}$ dependence of both $\varepsilon$ and the skin depth $\delta$, and $f_{0}, f_{1}$ being the two first Fourier terms of the groove series expansion]. Expression (1) reproduces the asymmetrical gap broadening, ${ }^{35}$ i.e., the lower band edge depends more strongly on changes in the plasma frequency. This behavior is in agreement with the measurements that show that switching occurs only near the lower frequency edge.

\section{B. Temperature dependence of the switching}

To investigate the dependence of the optical switch on temperature we have measured the transmission of SPPs through the grating with a fixed pump power density $\left(7 \mathrm{~W} / \mathrm{cm}^{2}\right)$, at different grating temperatures. These differential transmissions are represented in Fig. 5. The triangles, squares, and circles correspond to temperatures of $+20,0$, and $-35^{\circ} \mathrm{C}$, respectively. The switching efficiency is the largest when the grating temperature is $-35^{\circ} \mathrm{C}$ and decreases strongly at higher temperatures. From the fits to the measurements of Fig. 5 we obtain $\overline{\nu_{p}}=2.3,1.7$, and $1.4 \mathrm{THz}$ for $+20,0$, and $-35{ }^{\circ} \mathrm{C}$, respectively. The average carrier 


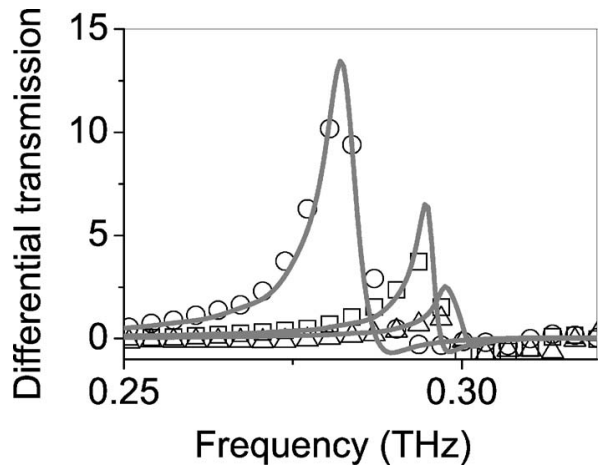

FIG. 5. Differential SPP transmission through a grating of InSb at different temperatures illuminated by a laser beam with a diameter of $1 \mathrm{~cm}, \lambda=1075 \mathrm{~nm}$, and power density $7 \mathrm{~W} / \mathrm{cm}^{2}$. The triangles correspond to a temperature of $+20{ }^{\circ} \mathrm{C}$, and the squares and circles to 0 and $-35^{\circ} \mathrm{C}$, respectively. The solid lines are fits to the measurements.

density derived from $\overline{\nu_{p}}$ is $\bar{N}=(1.4,0.8$, and 0.5$)$ $\times 10^{16} \mathrm{~cm}^{-3}$ for $+20,0$, and $-35^{\circ} \mathrm{C}$. The optical switching efficiency is determined by the ratio between optically and thermally excited carriers. For a fixed pump power density this ratio is larger at low temperatures due to the reduced number of thermally excited carriers, leading to a more efficient switch as it is apparent in Fig. 5.

\section{DYNAMICAL SWITCHING}

At this point it is interesting to discuss the time scales involved in the optical switching process. The time scale over which the switching occurs is determined by the optical injection of carriers in semiconductors, which takes place on the subpicosecond time scale. ${ }^{36-38}$ A pump-probe calculation is shown in Fig. 6 in the case of a Gaussian pump beam centered at $\lambda=1075 \mathrm{~nm}$ with a $1 / e$ width of $1 \mathrm{ps}$, and four probe beams centered at $\lambda=993 \mu \mathrm{m}(\nu=0.302 \mathrm{THz})$ and of widths $100 / \pi$ (solid line), $100 /(3 \pi)$ ps (dashed line), $10 / \pi$

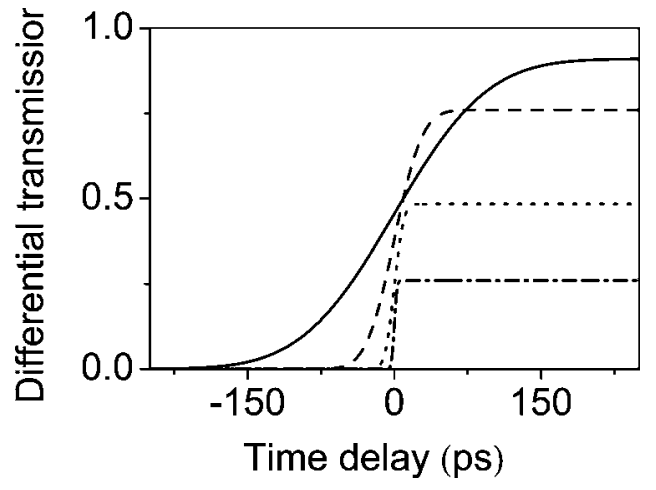

FIG. 6. Calculation of the differential SPP transmission through the InSb grating of Fig. 1(a) (at $-35^{\circ} \mathrm{C}$ ), as a function of the pump-probe time delay for a Gaussian pump beam centered at $\lambda$ $=1075 \mathrm{~nm}$ with a $1 / e$ width at half maximum of $1 \mathrm{ps}$. Four Gaussian probe beams are considered centered at $\lambda=993 \mu \mathrm{m}(\nu$ $=0.302 \mathrm{THz})$ of widths $100 / \pi$ (solid line), $100 /(3 \pi)$ (dashed line), $10 / \pi$ (dotted line), and $10 /(3 \pi)$ ps (dash-dotted line). (dotted line), and $10 /(3 \pi)$ ps (dash-dotted line). In this figure, the differential SPP transmission is represented as a function of the time delay between the pump at $0 \mathrm{ps}$ and the maximum intensity of the probe pulse. This differential transmission is defined as $\left(T-T_{0}\right) / T_{\max }$, where $T$ is the transmission at a certain time delay, $T_{0}$ is the transmission for a negative time delay larger than the duration of the probe pulse, and $T_{\max }$ is the transmission for a positive time delay larger that the duration of the probe pulse but smaller than the carrier recombination lifetime. The plateau at positive time delays in Fig. 6 has been imposed in the calculations on the basis of a $1 \mathrm{~ns}$ carrier recombination lifetime. ${ }^{36}$ The rise time of the differential transmission is governed by the probe beam widths, since the carrier excitation times are much shorter. Recall that this rise time can be brought down to the ps range provided that the switching spectral range is wider; in light of Eq. (1), this could be achieved by fabricating groove arrays with smaller periods, exhibiting the first gap at higher frequencies (closer to the $\nu_{p}$ ). A large switching efficiency, i.e., $\left(T-T_{0}\right) / T_{\max }$ close to one at large time delays, is only obtained for the longer pulse due to the fact that the frequency range within which the SPP gap edge shifts, i.e., the switching range, is narrow (see Fig. 3). The bandwidth of shorter pulses is larger than the switching range, diminishing the switching efficiency. Based on Fig. 6, we can conclude that semiconductor structures will allow ultrafast active plasmonic components at repetition rates determined by the carriers lifetime. Moreover, the low optical power levels required for carrier injection, will permit switching at ultra-low optical fluences, i.e., lower than $\mu \mathrm{J} / \mathrm{cm}^{2}$.

\section{CONCLUSIONS}

We have demonstrated the optical switching of the inplane transmission of SPPs through a Bragg mirror or grating of grooves structured on InSb. The switching is due to the modification of the semiconductor permittivity and the SPPs characteristics induced by the optical injection of free carriers. The switching depends on the optical pump power and wavelength as well as on the temperature of the grating. Optical power densities as low as $7 \mathrm{~W} / \mathrm{cm}^{2}$ at $\lambda=1075 \mathrm{~nm}$ give a 13-fold increase of the differential transmission through a grating at $-35^{\circ} \mathrm{C}$. Since the dynamics of the switching is determined by the excitation and relaxation of free carriers a semiconductor, ultrafast switchable mirrors for SPPs at extremely low optical fluences $\left(\mu \mathrm{J} / \mathrm{cm}^{2}\right)$ are feasible.

\section{ACKNOWLEDGMENTS}

We gratefully acknowledge B. Hadam for making the SEM photograph and the financial support from the European Union through the projects Interaction (Grant No. HPRN-CT-2002-00206) and Metamorphose (Grant No. NMP3-CT-2004-500252), and from the Deutsche Forschungsgemeinschaft. The work of J.G.R. was supported in part by the "Stichting voor Fundamenteel Onderzoek der Materie (FOM)," which is financially supported by the "Nederlandse Organisatie voor Wetenschappelijk Onderzoek 
(NWO)." The work of J.A.S.G. was supported in part by the Spanish Comunidad de Madrid (Grants No. GR/MAT/0425/
2004 and MICROSERES) and MEC (Grants No. FIS200607894 and No. FIS2004-0108).
${ }^{1}$ H. Raether, Surface Plasmons (Springer-Verlag, Berlin, 1988).

${ }^{2}$ J. Takahara and T. Kobayashi, Opt. Photonics News 275, 54 (2004) and references therein.

${ }^{3}$ S. Nie and S. R. Emory, Science 275, 1102 (1997).

${ }^{4}$ S. I. Bozhevolnyi and F. A. Pudonin, Phys. Rev. Lett. 78, 2823 (1997).

${ }^{5}$ T. W. Ebbesen, H. J. Lezec, H. F. Ghaemi, T. Thio, and P. A. Wolff, Nature (London) 391, 667 (1998).

${ }^{6}$ J. R. Krenn et al., Europhys. Lett. 60, 663 (2002).

${ }^{7}$ W. L. Barnes, A. Dereux, and T. W. Ebbesen, Nature (London) 424, 824 (2003) and references therein.

${ }^{8}$ P. Andrew and W. L. Barnes, Science 306, 1002 (2004).

${ }^{9}$ A. V. Krasavin, K. F. MacDonald, N. I. Zheludev, and A. Zayats, Appl. Phys. Lett. 85, 3369 (2004).

${ }^{10}$ T. Nikolajsen, K. Leosson, and S. I. Bozhevolnyi, Appl. Phys. Lett. 85, 5833 (2004).

${ }^{11}$ S. Park and S. H. Song, Electron. Lett. 42, 402 (2006).

${ }^{12}$ Y. R. Leroux, J. C. Lacroix, K. I. Chane-Ching, C. Fave, N. Félidj, G. Lévi, J. Aubard, J. R. Krenn, and A. Hohenau, J. Am. Chem. Soc. 127, 16022 (2005).

${ }^{13}$ I. I. Smolyaninov, A. V. Zayats, A. Gungor, and C. C. Davis, Phys. Rev. Lett. 88, 187402 (2002).

${ }^{14}$ I. I. Smolyaninov, A. V. Zayats, A. Stanishevsky, and C. C. Davis, Phys. Rev. B 66, 205414 (2002).

${ }^{15}$ J. Dintinger, I. Robel, P. V. Kamat, C. Genet, and T. W. Ebbesen, Adv. Mater. (Weinheim, Ger.) 18, 1645 (2006).

${ }^{16}$ J. A. Porto, L. Martín-Moreno, and F. J. García-Vidal, Phys. Rev. B 70, 081402(R) (2004).

${ }^{17}$ J. Gómez Rivas, P. Haring-Bolívar, and H. Kurz, Opt. Lett. 29, 1680 (2004).

${ }^{18}$ C. Janke, J. Gómez Rivas, P. Haring-Bolívar, and H. Kurz, Opt. Lett. 30, 2357 (2005).

${ }^{19}$ J. Gómez Rivas, J. A. Sánchez Gil, M. Kuttge, P. Haring Bolivar, and H. Kurz, Appl. Phys. Lett. 88, 082106 (2006).

${ }^{20}$ J. A. Sánchez-Gil and J. Gómez Rivas, Phys. Rev. B 73, 205410 (2006).

${ }^{21}$ J. B. Pendry, A. J. Holden, W. J. Stewart, and I. Youngs, Phys. Rev. Lett. 76, 4773 (1996).
${ }^{22}$ J. B. Pendry, L. Martin-Moreno, and F. J. Garcia-Vidal, Science 305, 847 (2004).

${ }^{23}$ A. P. Hibbins, B. R. Evans, and J. R. Sambles, Science 308, 670 (2005).

${ }^{24}$ S. J. Allen, D. C. Tsui, and R. A. Logan, Phys. Rev. Lett. 38, 980 (1977).

${ }^{25}$ J. Saxler, J. G. Rivas, C. Janke, H. P. M. Pellemans, P. Haring Bolivar, and H. Kurz, Phys. Rev. B 69, 155427 (2004).

${ }^{26}$ E. V. Alieva, G. Beitel, L. A. Kuzik, A. A. Sigarev, V. A. Yakovlev, G. N. Zhizhin, A. F. G. van der Meer, and M. J. van derWiel, Appl. Spectrosc. 51, 584 (1997).

${ }^{27}$ J. Gómez Rivas, M. Kuttge, P. Haring Bolivar, H. Kurz, and J. A. Sánchez-Gil, Phys. Rev. Lett. 93, 256804 (2004).

${ }^{28}$ J. Gómez Rivas, C. Janke, P. Haring Bolivar, and H. Kurz, Opt. Express 13, 847 (2005).

${ }^{29}$ I. I. Smolyaninov, D. L. Mazzoni, J. Mait, and C. C. Davis, Phys. Rev. B 56, 1601 (1997).

${ }^{30}$ J. A. Sánchez-Gil and A. A. Maradudin, Appl. Phys. Lett. 86, 251106 (2005).

${ }^{31}$ T. Sondergaard and S. I. Bozhevolnyi, Phys. Rev. B 69, 045422 (2004).

${ }^{32}$ J. A. Sánchez-Gil and M. Nieto-Vesperinas, J. Opt. Soc. Am. A 8, 1270 (1991).

${ }^{33}$ J. A. Sánchez-Gil, Phys. Rev. B 53, 10317 (1996).

${ }^{34}$ The calculations reproduce very accurately the width and the intensity of the peak measured in the differential transmission. However, the position of this peak was systematically displaced by approximately a $6 \%$ to higher frequencies, probably due to uncertainties in the depth of the grooves or imperfections in their shape. This shift is corrected in Figs. 3 and 5.

${ }^{35}$ S. C. Kitson, W. L. Barnes, and J. R. Sambles, Phys. Rev. Lett. 77, 2670 (1996).

${ }^{36}$ S. W. Leonard, H. M. van Driel, J. Schilling, and R. B. Wehrspohn, Phys. Rev. B 66, 161102(R) (2002).

${ }^{37}$ V. R. Almeida, C. A. Barrios, R. R. Panepucci, and M. Lipson, Nature (London) 431, 1081 (2004).

${ }^{38}$ A. Liu et al., Nature (London) 427, 615 (2004). 